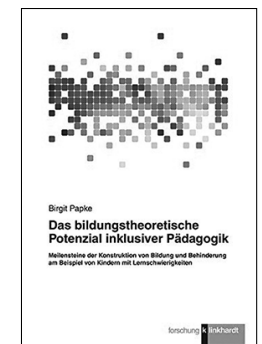

Papke, Birgit (2016):

Das bildungstheoretische Potenzial inklusiver Pädagogik. Meilensteine der Konstruktion von Bildung und Behinderung am Beispiel von Kindern mit Lernschwierigkeiten

Bad Heilbrunn: Klinkhardt. 210 S., € 39,-

Seit dem Inkrafttreten der UN-Konvention über die Rechte von Menschen mit Behinderung wird allenthalben über inklusive Bildung gesprochen. Aber bislang liegen nur sehr spärliche Versuche im deutschsprachigen Raum vor, dies begrifflich und theoretisch zu klären. Genau mit dieser Frage hat sich Birgit Papke dankenswerter Weise in ihrer Dissertation beschäftigt. Nach einem Vorwort der Doktormutter, Maria Kron, und den einleitenden Überlegungen widmet sich Frau Papke dem Zusammenhang von Bildung und Behinderung (Kap. 2). In Kap. 3 erläutert sie ihre forschungsmethodische Vorgehensweise, die sie im Wesentlichen über eine umfangreiche Literaturrecherche zum Thema inklusive Bildung und eine darauf bezogene Dokumentenanalyse gestaltet. Dabei ist sie sowohl an der konzeptionellen Ebene der Leitideen als auch an der disziplinären Ebene der beteiligten Teildisziplinen der Erziehungs- und Bildungswissenschaften interessiert. Das Kap. 4 enthält einen sehr lesenswerten Gang durch die Geschichte des Begriffs Bildung und den Wandel der Bildungsvorstellungen. Hier wird bereits deutlich, dass sich bis heute nur wenige Bildungstheorien tatsächlich um eine Einbeziehung von Kindern, Jugendlichen und Erwachsenen mit Behinderung bemüht haben. In Kap. 5, 6 und 7 stellt die Autorin die Entwicklung der Bil- 
dungsvorstellungen in der Heil- und Sonderpädagogik dar, wobei sie sich konzentriert auf den Förderschwerpunkt geistige Entwicklung, nicht ganz eindeutig als „Menschen mit Lernschwierigkeiten" bezeichnet. In Kap. 8 und 9 stellt die Autorin den Wandel der Bildungsvorstellungen von der Phase der Integration zur Phase der Inklusion dar. Hier ist besonders hervorzuheben, dass sie dabei nicht nur den historischen Rückblick anstrebt, sondern auch neuere konstruktivistische Positionen mit aufnimmt. Im Ergebnis stellt die Autorin Bildung nicht nur als Prozess, sondern ebenso als Konstruktion und Transformation dar. Damit zeigt sie eindrücklich auf, dass in einer inklusiven Bildungskonzeption nicht nur eine begrenzte Erweiterung unseres Bildungsverständnisses ansteht, sondern vielmehr eine Neubestimmung dessen, was wir zukünftig unter Bildung im Zeitalter der Inklusion verstehen wollen. Damit ist letztlich das „bildungstheoretische Potenzial inklusiver Pädagogik“ erschlossen, auch wenn neben den zahlreichen Erkenntnissen innerhalb des Bandes abschließend ebenso die offenen Baustellen nicht unerwähnt bleiben. Das Buch von Birgit Papke stellt einen substanziellen Beitrag zum Diskurs über inklusive Bildung dar, der sowohl einseitige ideologische Verkürzungen vermeidet als auch trügerische Gewissheiten aufdeckt.

\section{Prof. Dr. Ulrich Heimlich}

D-80802 München

DOI 10.2378/vhn2018.arto8d 